\title{
Geometric Calibration of Focused Light Field Camera For 3-D Flame Temperature Measurement
}

\author{
Jun Sun ${ }^{1}$, Chuanlong Xu ${ }^{1 *}$, Biao Zhang ${ }^{1}$, Shimin \\ Wang \\ ${ }^{1}$ School of Energy and Environment, Southeast University \\ 230149021@seu.edu.cn, chuanlongxu@seu.edu.cn, \\ zhangbiao@seu.edu.cn,101002400@seu.edu.cn
}

Hong $\mathrm{Qi}^{3}$ and Heping $\operatorname{Tan}^{3}$

${ }^{3}$ School of Energy Science and Engineering, HIT, West Dazhi Street, Harbin, PR China qihong@ hit.edu.cn, tanheping@ hit.edu.cn
Md. Moinul Hossain ${ }^{2}$

${ }^{2}$ Department of Chemical and Process Engineering, University of Strathclyde, UK. m.hossain@ strath.ac.uk

\begin{abstract}
Focused light field camera can be used to measure three-dimensional (3-D) temperature field of a flame because of its ability to record intensity and direction information of each ray from flame simultaneously. This work aims to develop a suitable geometric calibration method of focused light field camera for 3-D flame temperature measurement. A modified method based on Zhang's camera calibration is developed to calibrate the camera and the measurement system. A single focused light-field camera is used to capture images of bespoke calibration board instead of flame images in this study. Geometric parameters including intrinsic (i.e., camera parameters) and extrinsic (i.e., camera connecting with the calibration board) of the focused light field camera are calibrated to trace the ray projecting onto each pixel on CCD (chargecoupled device) sensor. Instead of using line features, corner point features are directly utilized for the calibration. The characteristics of focused light field camera including one 3-D point corresponding to several image points and matching main lens and microlens f-numbers, are used for calibration. Results with a focused light field camera are presented and discussed. Preliminary the 3-D temperature distribution of a flame is also investigated and presented.
\end{abstract}

Keywords-Focused light field camera; Zhang's calibration method; camera calibration; ray tracing

\section{INTRODUCTION}

Light field camera can record the direction of each ray projected onto the sensor. So the direction of each ray emitted by flame with corresponding intensity could be simultaneously obtained through a light field camera. A single focused light field camera can be used to measure 3-D temperature field of a flame in combination with reconstruction algorithms. The schematic diagram of radiative formation model of a single light field camera is illustrated in Fig.1. In Fig. 1, $L$ is the distance between main lens and microlens array, $l_{m}$ is the distance between sensor plane and microlens array and $S_{v}$ is the distance between virtual image plane and microlens array. The focal plane of the light field camera applied to capture translucent medium (flame), is called virtual focal plane. The point on virtual focal plane is called the virtual source point. The virtual image plane is the conjugate plane of the virtual

This work is supported by Special Fund for Basic Research on Scientific Instruments of the National Natural Science Foundation of China (No. 51327803) and the Natural Science Foundation of Jiangsu Province for Distinguished Young Scholars (No. BK20150023). focal plane for the main lens. The point on virtual image plane is called the virtual image point. The rays emitted by one virtual source point are converged to the virtual image point by main lens and then reconverged to the pixels (i.e. image points) of the sensor by the microlens in the focused light field camera [1].

Since the cone angle of the beam projecting onto one pixel is very small (less than $0.1^{\circ}$ at the diameter of main lens aperture of $4 \mathrm{~mm}$ and distance between the camera and virtual focal plane of $270 \mathrm{~mm}$ ), the principal ray, which crosses through the pixel and the center of its corresponding microlens, is used to represent the beam of rays projecting onto the pixel. It is called the corresponding ray of the pixel. The intensity detected by the pixel is the intensity of the corresponding ray from the flame. In recent years, various techniques were proposed to reconstruct the 3-D temperature distribution from known radiative intensity of the flames [2][3]. For instance, Li et al. [2] proposed a radiative imaging model based on conventional CCD cameras for flame temperature measurement. The 3-D flame temperature field is reconstructed using a Tikhonov regularization method. Niu et al. [3] employed a hybrid LSQR-PSO (least-square QR decomposition-particle swarm optimization) algorithm to estimate the 3-D temperature distributions and absorption coefficient simultaneously with known radiative intensity of the flame detected by a hypothetical plane. Several multicameras based tomographic and laser based diagnostics techniques were also reported to reconstruct the 3-D temperature distribution of the flames [4-7]. However, multicameras techniques are in high system cost, complexity in system setup and installation and laser based diagnostics techniques require more complex system and unsuitable for industrial furnaces due to the complex setup, high cost of the system setup.

The 3-D temperature reconstruction method used in this study is based on the techniques proposed in [2][3]. The relationship between the intensity of each ray from the flame and the intensity detected by the sensor can be established by the radiation transfer equations and the model for radiative image formation of the focused light field camera [8]. The radiation intensity of each control volume of the flame is then obtained by solving the equations and hence 3 -D temperature 


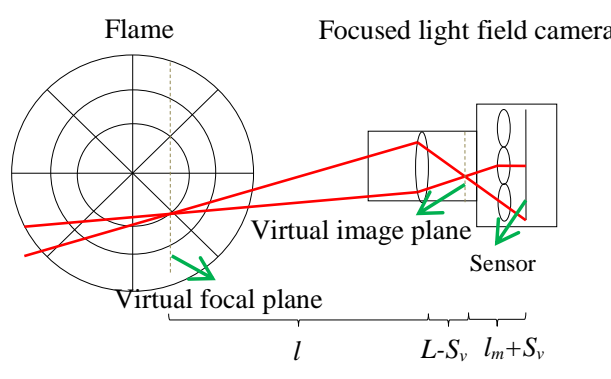

Fig. 1. Schematic diagram of radiative formation model of focused light field camera

field of the flame can be derived through use of Planck's radiation law [9].

In the radiative image formation model, it is crucial to trace the corresponding ray of the pixel accurately and to determine its direction and position outside the camera. Normally, the rays can be traced from one lens to next lens using thin lens equations based on the simple pinhole model [9]. So $L, l_{m}, S_{v}$ and coordinate transformation matrix $[\mathbf{R}, \mathbf{t}]$ from the camera coordinate to the world coordinate must be determined through calibration to trace the rays [10].

For conventional camera, Zhang's calibration method based on pinhole camera model is usually utilized to determine the relationship between $3-\mathrm{D}$ point in calibration board and the image point on the sensor plane [12]. However, because the rays are converged twice by the main lens and microlens in the focused light field camera, Zhang's calibration method cannot be employed directly to calibrate the focused light field camera. Yunsu Bok developed an efficient geometric calibration method for traditional light field camera (i.e. lytro lightfield camera) using line features [13]. However, the distance between the sensor plane and the microlens array in the lytro light field camera is equal to the focal length of each microlens. Hence the 3-D point in calibration board is not imaged directly on the sensor. It is therefore difficult to extract precise locations of the corner points from raw images captured by the lytro light field camera. To fully capture the positional information of the light field, the microlenses are focused on the image produced by the main lens in the focused light field camera [14]. So in the focused light field camera the corner points are imaged on virtual image plane by main lens and then re-imaged on the CCD sensor by the microlenses. The points are thus recognizable on the raw image of calibration board. As shown in Fig. 2, the corner point (marked by red circle) in the image captured by a focused light field camera is recognizable to be picked up, while there are no recognizable corner points in the image captured by a traditional light field camera. The recognized corner points can then be used for the calibration. So the line features are not necessary for the geometric calibration of the focused light field camera.

The method proposed by Yunsu Bok cannot determine all the parameters $\left(L, l_{m}, S_{v}\right.$ and $\left.[\mathbf{R}, \mathbf{t}]\right)$. Ole et al. proposed a calibration method for focused light field camera [15]. Sequential quadratic programming (SQP) algorithm was employed to optimize the residual which depends on the unknown parameters of the light field camera. However, a

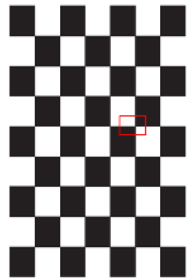

(a) Calibration board

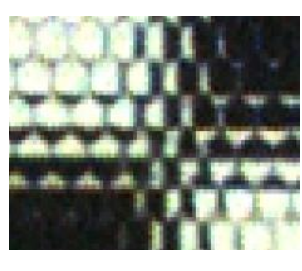

(b) Traditional Light field camera

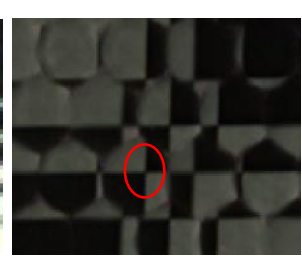

(c) Focused Light field camera
Fig. 2. Images of calibration board captured by traditional and foucused light field cameras

good initialization is required for the accurate optimization or the algorithm may be converged to local optima.

In this study, the distance ratio $l_{m} / S_{v}$, is calculated using the coordinate of one virtual image point and two image points on CCD sensor [refer to Fig. 1]. Meanwhile, the same f-numbers of main lens and microlens are applied to establish the calibration equations. This paper aims to propose a modified method for the calibration of the focused light field camera based on Zhang's method [13]. The principle of the calibration method is presented in details. Calibration experiments of a focused light field camera are performed by using a bespoke calibration board. The results are presented and discussed as well.

\section{MODIFIED CALIBRATION METHOD}

The center of the microlens must be known for the modified calibration method. In this study, it can be determined by analyzing white-plane image. The center coordinate of the sub-images covered by microlenses are computed by taking following three steps [16]: (a) Binaryzation of the raw whiteplane images with a proper threshold value which depends on the illumination intensity of the white-plane image. Generally, the gray level of the outermost pixel of the central sub-image can be used as the threshold, $(b)$ determination of the edge of each sub-image using the binarized image and $(c)$ calculation of the center of each sub-image according to following equations

$$
x_{k}=\frac{\sum_{(i, j) \in J_{k}} j \cdot I(i, j)}{\sum_{(i, j) \in J_{k}} I(i, j)}
$$

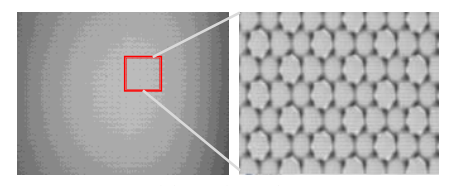

(a) White-plane image

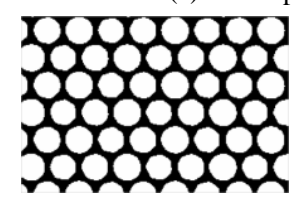

(b) Binarized image

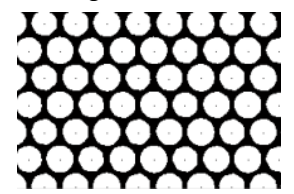

(c) Center of microlens

Fig. 3. Determination of center of microlens in focused light field camera 


$$
y_{k}=\frac{\sum_{(i, j) \in J_{k}} i \cdot I(i, j)}{\sum_{(i, j) \in J_{k}} I(i, j)}
$$

where $k$ denotes the $k^{\text {th }}$ microlens, $x$ and $y$ denote the center coordinate of the $k^{\text {th }}$ sub-image, $J_{k}$ denotes the coordinate of all the pixels of the $k^{\text {th }}$ sub-image, and $\mathrm{i}$ and $\mathrm{j}$ denote the coordinate of each pixel of the $k^{\text {th }}$ sub-image. $I(i, j)$ denotes the gray level of the corresponding pixel in the binarized image.

Through processing the white-plane image, the center of each sub-image can be determined accurately, as shown in Fig. 3. The principal plane of the microlens and the CCD sensor plane are parallel, so each microlens has the same central coordinate with each sub-image.

Zhang' method relies on common pinhole camera model. The relationship between a 3-D point $\mathbf{M}$ and its image projection $\mathbf{m}$ is given by

$$
s \tilde{\mathbf{m}}=\mathbf{A}[\mathbf{R} \mathbf{t}] \tilde{\mathbf{M}}
$$

where $s$ is an arbitrary scale factor, and the extrinsic parameters $(\mathbf{R}, \mathbf{t})$ is the rotation and translation vectors which relates the world coordinate to the camera coordinate. The intrinsic matrix $\mathbf{A}$ is given by

$$
\mathbf{A}=\left[\begin{array}{llc}
\alpha & \gamma & u_{0} \\
0 & \beta & v_{0} \\
0 & 0 & 1
\end{array}\right]
$$

where $\left(u_{0}, v_{0}\right)$ is the coordinate of the principal point of the lens, $\alpha$ and $\beta$ are the scale factors of $u$ and $v$ axes of the image, and $\gamma$ is the parameter describing the skewness of the two image axes.

The focused light field camera consists of two layers of lenses: the main lens and a microlens array. The 'pinhole camera model' is applied for the lenses. Since the virtual image plane and the virtual focal plane are conjugated for the main lens, the virtual image point $\left(\mathbf{m}^{\prime}\right)$ and the corresponding 3-D point $(\mathbf{M})$ are also conjugated. Therefore, the virtual image point ( $\mathbf{m}$ ') is required instead of $\mathbf{m}$ when Zhang's method is applied to calibrate the focused light field camera. As shown in Fig. 4, $x, M_{x}, V_{x}$ and $X_{c}$ are the $x$ coordinate of the image point $(\mathbf{m})$, center of the corresponding microlens, virtual image point (m') and 3-D point (M) in camera coordinate system, respectively. Each 3-D point $(\mathbf{M})$ is located on the focal plane of the focused light field camera, according to pinhole model, the relationship among $V_{x}, M_{x}$ and $x$ is given by

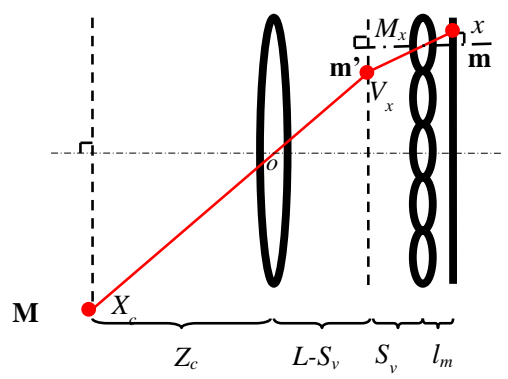

Fig. 4. Schematic diagram of the focused light field camera model

$$
\frac{l_{m}}{S_{v}}=-\frac{x-M_{x}}{V_{x}-M_{x}}
$$

The relationship between $V_{x}$ and $X_{c}$ is expressed by

$$
\frac{L-S_{v}}{Z_{c}}=-\frac{V_{x}}{X_{c}}
$$

Equation (7) can be derived through solving (5) and (6),

$$
V_{x}=\frac{S_{v}}{l_{m}}\left(x-\left(1+\frac{l_{m}}{S_{v}}\right) M_{x}\right)=-\frac{\left(L-S_{v}\right)}{Z_{c}} X_{c}
$$

The image formated by the main lens as shown in Fig. 4 is displayed upside down from observed persperctive. Equation (7) can be converted into (8) by adding minus to $x$ and $M_{x}$.

$$
V_{x}=-\frac{S_{v}}{l_{m}}\left(x-\left(1+\frac{l_{m}}{S_{v}}\right) M_{x}\right)=\frac{\left(L-S_{v}\right)}{Z_{c}} X_{c}
$$

According to (8), the relationship between a 3-D point (M) and its image projection (m') is given by

$$
\begin{gathered}
s \tilde{\mathbf{m}}^{\prime}=\mathbf{A}[\mathbf{R} \mathbf{t}] \tilde{\mathbf{M}} \\
\tilde{\mathbf{m}}^{\prime}=\left[-\frac{S_{v}}{l_{m}}\left(x-\left(1+\frac{l_{m}}{S_{v}}\right) M_{x}\right),-\frac{S_{v}}{l_{m}}\left(y-\left(1+\frac{l_{m}}{S_{v}}\right) M_{y}\right), 1\right]^{T} \\
\tilde{\mathbf{M}}=[X, Y, Z, 1]^{T}
\end{gathered}
$$

where $(X, Y, Z)$ is the coordinate of $3-\mathrm{D}$ point $(\mathbf{M})$ in world coordinate system. Note that different from those of conventional camera, the parameters $\alpha, \beta$ and $\gamma$ of $\mathbf{A}$ in (4) are decribed as

$$
\begin{gathered}
\alpha=\frac{L-S_{v}}{d x} \\
\beta=\frac{L-S_{v}}{d x} \\
\gamma=\frac{\left(L-S_{v}\right) \tan a}{d y}
\end{gathered}
$$

where $d x$ and $d y$ are the sizes of the pixel of the CCD sensor and $a$ denotes the angle of skewness of two image axes.

The distance $l_{m} / S_{v}$ ratio, defined as $\beta_{m}$, is needed to determine the unknown point $\mathbf{m}$ ' in the virtual image from the image point $\mathbf{m}$ on the sensor plane by using (10). As the CCD sensor is placed at a distance which is unequal to $f_{m}$ (focal length of microlens) in the focused light field camera, the 3-D points in the calibration board are imaged at distance $S_{v}$ in front of the microlenses. The virtual image is further projected onto pixels on the CCD sensor by the microlenses. One virtual image point of calibration the board is then projected onto several image points on the sensor plane by the microlenses array. Two different image points on the sensor plane and their corresponding virtual image point were utilized to calculate $l_{m} / S_{v}$. As shown in Fig.5, $V_{u}, u_{1}, u_{2}, M_{u 1}$ and $M_{u 2}$ are $u$ coordinate of virtual image point $(u, v)$, image point $\mathbf{m}_{\mathbf{1}}$, image 
point $\mathbf{m}_{2}$, center of the microlens corresponding to $\mathbf{m}_{1}$ and center of the microlens corresponding to $\mathbf{m}_{\mathbf{2}}$ in image coordinate system, respectively. Note that $u_{1}$ and $u_{2}$ must be not equal, and so are $M_{u 1}$ and $M_{u 2}$. Similar to (8), the relationship is described as

$$
V_{u}=\frac{S_{v}}{l_{m}}\left(u_{1}-\left(1+\frac{l_{m}}{S_{v}}\right) M_{u 1}\right)=\frac{S_{v}}{l_{m}}\left(u_{2}-\left(1+\frac{l_{m}}{S_{v}}\right) M_{u 2}\right)
$$

By solving (15), $\beta_{m}$ can be obtained,

$$
\beta_{m}=\frac{l_{m}}{S_{v}}=\frac{u_{1}-u_{2}}{M_{u 1}-M_{u 2}}-1
$$

where $u_{1}$ and $u_{2}$ are known directly from the raw image of calibration board, $M_{u 1}$ and $M_{u 2}$ are also known according to (1) and (2).

Several images (usually greater than 3) of calibration board must be captured to solve (9). However, the aim of the calibration of the focused light field camera in this paper is to measure 3-D flame temperature, so the world coordinate system is based on the burner location. The center of the outlet of the burner is regarded as the origin of the world coordinate system. $\mathrm{x}$ and $\mathrm{y}$ axis in the system is the $\mathrm{x}$ and $\mathrm{y}$ axis of calibration board fixed on the burner, and $\mathrm{z}$ axis is normal to the calibration board. In the camera coordinate system, the principal point of the main lens is taken as the origin, $\mathrm{x}$ and $\mathrm{y}$ axis are parallel to image plane, and $\mathrm{z}$ axis is normal to image plane. Once several images of the calibration board under different orientations by moving the plane are captured by the focused light field camera, the five intrinsic parameters $(\alpha, \beta, \gamma$, $u_{0}$ and $v_{0}$ ) and four extrinsic parameters (including three rotation transformation vectors $\mathbf{r}_{1}, \mathbf{r}_{2}, \mathbf{r}_{3}$ and one translation transformation vector $\mathbf{t}$ ) can be determined by solving (9). Therefore $L-S_{v}$ can be calculated using (12-13) with known $\alpha, \beta$, $d x$ and $d y$.

To obtain the $l_{m}, S_{v}$ and $L$ further, the matching f-numbers of the main lens and microlens of the focused light field camera has to be utilized [17], as shown in Fig. 6. Relative sizes of the main lens and microlens apertures are optimized so that the sub-images under each microlens are as large as possible, and have no overlapping in the focused light field camera. In this case the f-numbers of the main lens and microlens are equal when the sub-images (regarded as circles) are tangential. Note that the f-number of the lens refers to the image-side f-number, and so the f-number of the main lens is the ratio of the distance

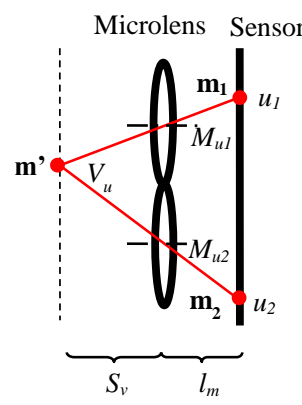

Fig. 5. Schematic diagram of calculating $\beta_{m}$ using two image points on sensor plane and their corresponding virtual image point
$L$ to diameter of main lens aperture and the f-number of the microlens is the ratio of the distance $l_{m}$ to diameter of microlens aperture. The matching f-number is described as

$$
\frac{L}{D}=\frac{l_{m}}{d}
$$

where $D$ and $d$ are the diameters of the main lens and microlens apertures, respectively. $D$ is known and $d$ can be calculated according to the diameter of the sub-image. Combining (12) and (16) with (17), $L, l_{m}$ and $S_{v}$ can be derived

$$
\begin{gathered}
l_{m}=\frac{\alpha d x d \beta_{m}}{D \beta_{m}-d} \\
S_{v}=l_{m} / \beta_{m} \\
L=S_{v}+\alpha d x
\end{gathered}
$$

To accomplish the geometric calibration of the focused light field camera, the following procedures are taken;

a) Take a few images of the calibration board under different orientations by moving the plane, including an image of the calibration board which is fixed on the burner;

b) Pick the corner points in the images;

c) Calculate $\beta_{m}$ of each corner point using (16) and transform m into m';

d) Estimate the intrinsic parameters and all the extrinsic parameters using the closed-form solution;

e) Calculate $l_{m}, S_{v}$ and $L$ using (18-20).

Ray tracing through each layer of lens (microlens, main lens) can be calculated using thin lens equations (21-22) to determine the direction and position of the ray outside the camera.

$$
\begin{gathered}
\frac{1}{s^{\prime}}+\frac{1}{s}=\frac{1}{f} \\
\frac{x^{\prime}}{x}=-\frac{s^{\prime}}{s}
\end{gathered}
$$

where $s^{\prime}$ and $s$ are the object distance and image distance of the lens respectively. $s$ ' is equal to $s_{v}$ and $s$ is equal to $l_{m}$ for microlenses. $s$ ' is equal to $l$ and $s$ is equal to $L-s_{v}$ for main lens. $f$ is the focal length of the lens. $x$ ' and $x$ are the coordinate of the object and image respectively. According to [7], a linear equations (23) regarding to all the rays projecting onto each

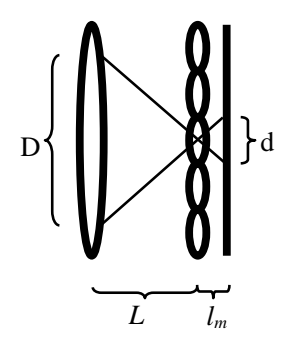

Fig. 6. Schematic diagram of calculating $\beta_{m}$ using two image points on sensor plane and their corresponding virtual image point 
pixel is derived and expressed by,

$$
\mathbf{I}_{\mathbf{c c d}}=\mathbf{A} \mathbf{I B}_{\lambda}
$$

where $\mathbf{I}_{\mathbf{c c d}}$ is the matrix of the intensity distribution on the CCD sensor, $\mathbf{I B}_{\lambda}$ is the matrix of the all voxels and can be calculated with the monochromatic intensity of blackbody radiation. $\mathbf{A}$ is the coefficient matrix. LSQR algorithm is used to solve (23) and to receive the monochromatic intensity of blackbody radiation $I_{b \lambda}$ of each voxel [18]. The temperature $T$ of each voxel is then calculated using (24) according to Planck's law.

$$
T=c_{2} / \lambda \ln \left[c_{1} /\left(\lambda^{5} \pi I_{b \lambda}+1\right)\right]
$$

where $c_{l}$ is the first radiation constant, $3.7418 \times 10^{-16} \mathrm{~W} \cdot \mathrm{m}^{2}$ and $c_{2}$ is the second radiation constant, $1.4388 \times 10^{-2} \mathrm{~m} \cdot \mathrm{K} . \lambda$ is the wavelength of the ray.

\section{RESULTS AND DISCUSSIONS}

In order to calibrate the camera, four images ((a)-(d)) of the calibration board are captured under different orientations (angles of board plane in respect to sensor plane) as shown in Fig. 7 and then the proposed method [section II] is applied. The resolution of the focused light field camera is $4384 \times 6576$ pixels and size of each pixel is $5.5 \times 5.5 \mu \mathrm{m}$. There are $22 \times 10$ corner points of calibration board utilized for the calibration. Table I summarizes the intrinsic parameters of the focused light field camera calibrated with four images. The calibrated value of $\mathrm{Sv}$ is negative indicating that the virtual image plane and CCD sensor plane are on the same side of the microlens array in the calibrated camera. Table II shows the extrinsic parameters corresponding to each image. Table III illuminates the calibration error. The error is calculated by the distances (in pixels) between the recognized image points and projected ones of 3-D points on calibration board. The mean errors of the images are less than 25 pixels. The calibration results indicated

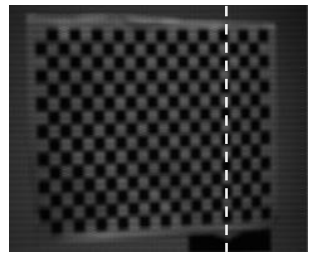

(a) $20^{\circ}$

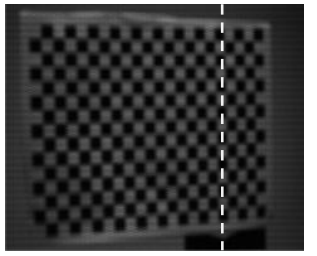

(c) $30^{\circ}$

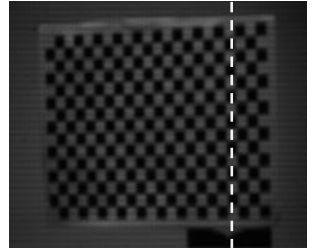

(b) $-20^{\circ}$

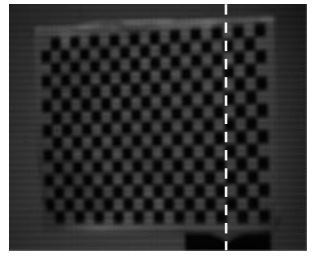

(d) $-10^{\circ}$
Fig.7. Images of calibration board catured by the focused lightfield camera under different orientations

TABLE I. INTRINSIC PARAMETERS OF THE FOCUSED LIGHT FIELD CAMERA

\begin{tabular}{|c|c|c|c|c|}
\hline $\boldsymbol{u}_{\boldsymbol{0}}$ (pixels) & $\boldsymbol{v}_{\boldsymbol{0}}$ (pixels) & $\boldsymbol{l}_{\boldsymbol{m}}(\mathbf{m m})$ & $\boldsymbol{S}_{\boldsymbol{v}}(\mathbf{m m})$ & $\boldsymbol{L}(\mathbf{m m})$ \\
\hline 3190.8 & 2545.1 & 0.592 .2 & -2.575 & 48.63 \\
\hline
\end{tabular}

that the proposed method is capable of calibrating the geometric features of a focused light field camera and the measurement system. The parameters of the focused light field camera are then applied to trace the rays using (21-22).

Preliminary 3-D temperature of ethylene flames is reconstructed and the results are shown in Fig. 8. The temperature distribution over each cross-sections of the flame is supposed to be axis-symmetric. However, the results shown in Fig. 8 are not axis-symmetric. This may be due to inaccurate

TABLE II. EXTRINSIC PARAMETERS OF THE FOCUSED LIGHT FIELD CAMERA

\begin{tabular}{|c|c|c|c|c|c|}
\hline Image & $\boldsymbol{t}(\boldsymbol{m m})$ & $\boldsymbol{r} \mathbf{l}$ & $\boldsymbol{r} \mathbf{c}$ & $\boldsymbol{r 3}$ & $\boldsymbol{s}(\boldsymbol{m m})$ \\
\hline \multirow{4}{*}{1} & -81.59 & 0.7636 & -0.5676 & -0.3183 & \\
& 93.40 & -0.6365 & -0.5850 & -0.4958 & 520.7 \\
& 520.7 & 0.0898 & 0.5825 & -0.8080 & \\
\hline \multirow{3}{*}{2} & -132.5 & 0.9833 & 0.1702 & -0.0948 & \\
& 85.98 & 0.0595 & -0.7602 & -0.6457 & 552.8 \\
& 552.8 & -0.1737 & 0.6266 & -0.7576 & \\
\hline \multirow{3}{*}{3} & -71.74 & 0.9901 & -0.0281 & 0.1358 & \\
& 91.35 & 0.0272 & -0.8824 & -0.4692 & 521.3 \\
& 521.3 & 0.1434 & 0.4701 & -0.8726 & \\
\hline \multirow{3}{*}{4} & 126.0 & 0.0880 & -0.9937 & -0.0746 & \\
& 92.39 & -0.9953 & -0.0889 & -0.0266 & 499.5 \\
& 499.5 & 0.0200 & 0.0767 & -0.9969 & \\
\hline
\end{tabular}

TABLE III.

ERRORS OF THE CALIBRATION RESULTS

\begin{tabular}{|c|c|c|c|}
\hline Image & $\begin{array}{c}\text { Mean error } \\
\text { (pixels) }\end{array}$ & $\begin{array}{c}\text { Maximum error } \\
\text { (pixels) }\end{array}$ & $\begin{array}{c}\text { RMS } \\
\text { (pixels) }\end{array}$ \\
\hline 1 & 5.89 & 34.27 & 7.56 \\
\hline 2 & 15.60 & 39.37 & 18.57 \\
\hline 3 & 9.97 & 21.85 & 11.10 \\
\hline 4 & 21.40 & 58.95 & 25.87 \\
\hline
\end{tabular}

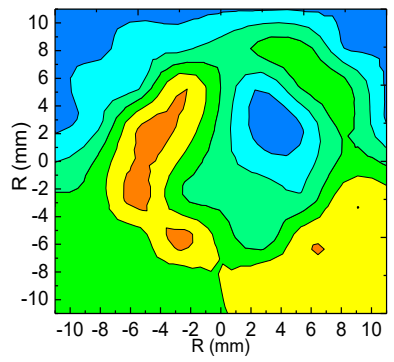

(a) $\mathrm{X}=6.67 \mathrm{~mm}$

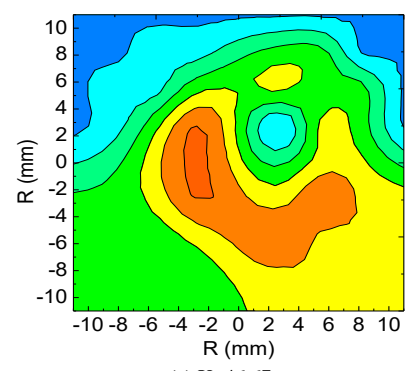

(c) $\mathrm{X}=46.67 \mathrm{~mm}$

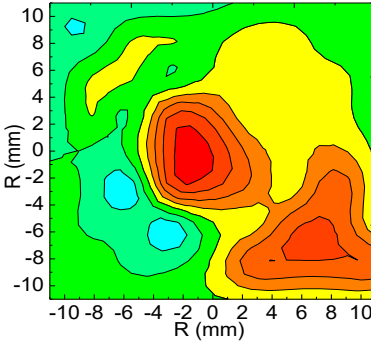

(b) $\mathrm{X}=33.33 \mathrm{~mm}$

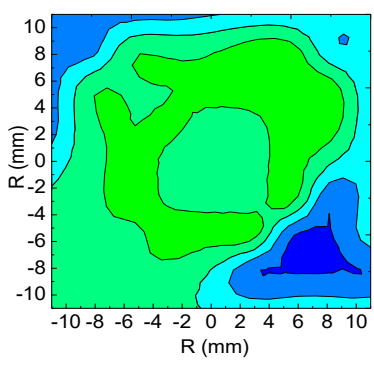

(d) $\mathrm{X}=73.33 \mathrm{~mm}$
Fig. 8. Reconstructed temperature distributions of the flame cross-sections 
direction and position of the traced ray outside the camera caused by the calibration error of the parameters $\left(l_{m}, s_{v}, L\right)$.

\section{CONCLUSIONS}

A modified geometric calibration method based on Zhang's method has been developed to calibrate the focused light field camera for the 3-D temperature measurement of a flame. The recognizable corner points of raw images of the calibration board and the centeres of microlenses have been identified. The geometric parameters of the focused light field camera have also been determined using the proposed method. The mean errors calculated by the distances (in pixels) between the recognized image points and projected ones of 3-D points on the calibration board are less than 25 pixels. The calibration results indicated that the modified proposed method is capable of calibrating the focused light field camera. The calibrated parameters of the focused light field camera have also been used to trace the ray projecting onto each pixel on CCD sensor. The 3-D temperature field of a ethylene flame have also been reconstructed. Results obtained from the experiments showed that the focused light field camera and the proposed method are capable of reconstructing 3-D flame temperature field. Future works will be focused on improving the calibration and reconstruction accuracy of the flame temperature measurement.

\section{REFERENCES}

[1] T. Georgiev and A. Lumsdaine, "Focused plenoptic camera and rendering," J. Electron. Imaging, Vol. 19, pp. 021106-1-021106-11, June 2010.

[2] W. Li, C. Lou, Y. Sun, and H. Zhou, "Estimation of radiative properties and temperature distributions in coal-fired boiler furnaces by a portable image processing system,” Exp. Therm. Fluid Sci, Vol 35, pp. 416-421, 2011.

[3] C. Niu, H. Qi, X. Huang, L. Ruan, W. Wang, and H. Tan, "Simultaneous reconstruction of temperature distribution and radiative properties in participating media using a hybrid LSQR-PSO algorithm," Chinese Phys. B, Vol 24, pp. 114401-1- 114401-11, 2015.

[4] Y. Yan, T. Qiu, G. Lu, M. M. Hossain, G. Gilabert and L. Shi, "Recent advances in flame tomography", Chin. J. Chem. Eng. vol. 20, pp. 38999, 2012.

[5] M. M. Hossain, G. Lu, D. Sun, and Y. Yan, "Three-dimensional reconstruction of flame temperature and emissivity distribution using optical tomographic and two-color pyrometric techniques," Meas. Sci. Technol, Vol 24, pp. 1-10, 2013.

[6] J. Doi and S. Sato, "Three-dimensional modeling of the instantaneous temperature distribution in a turbulent flame using a multidirectional interferometer," Opt. Eng, Vol 46, pp. 015601-1-015601-7, 2007.

[7] H. N. Yang, B. Yang, X. S. CAI, C. Hecht, T. Dreier, and C. Schulz, "Three-dimensional (3-D) temperature measurement in a low pressure flame reactor using multiplexed tunable diode laser absorption spectroscopy (TDLAS)," Laser. Eng, Vol 31, pp. 285-297, 2015.

[8] R. Viskanta, "Concerning the definitions of the mean absorption coefficient,” Int. J. Heat Mass Transfer, Vol. 7, pp. 1047-1049, 1964.

[9] M. Planck, The Theory of Heat Radiation," Courier Corporation, 1959.

[10] A. Fusiello, "Elements of geometric computer vision," Available fro m: http://homepages. inf. ed. ac. uk/ rbf/ CVonline/ LOCAL_COPIES /FUSIELLO4/ tutorial. html, 2006.

[11] S. Widnall, "Lecture 13-vectors, matrices and coordinate transformations, " Dynamics, pp. 1-15, 2009.

[12] Z. Zhang, "A flexible new technique for camera calibration," Pattern Analysis and Machine Intelligence, Vol. 22, pp. 1330-1334, 2000
[13] Y. Bok, H.G. Jeon, and I. S. Kweon, "Geometric calibration of microlens-based light-field cameras using line features," In Computer Vision-ECCV 2014, pp. 47-61, 2014.

[14] A. Lumsdaine and T. Georgiev, "The focused plenoptic camera," In Computational Photography (ICCP), 2009 IEEE International Conference on, pp. 1-8. IEEE, 2009.

[15] O. Johannsen, C. Heinze, B. Goldluecke, and C. Perwaß, "On the calibration of focused plenoptic cameras," Time-of-Flight and Depth Imaging, pp. 302-317, 2013.

[16] Z. Zhou and B. Xianglin, "Research on light field imaging technology," A dissertation for doctor degree of uinversity of science and technology of China, pp. 57-58, May 2012.

[17] R. Ng, M. Levoy, M. Brédif, G. Duval, M. Horowitz, and P. Hanrahan, "Light field photography with a hand-held plenoptic camera," Computer Science Technical Report CSTR 2, Vol. 11, pp. 1-11, 2005.

[18] C. Paige and M. Saunders, "LSQR: An algorithm for sparse linear equations and sparse least squares," ACM T. Math. Software, Vol. 8, pp. $43-71,1982$ 